\title{
Dynamic random network coding with variable multicast rate in DTN environment
}

\author{
XIA Yi \\ Institute of Traffic Information \\ Hubei Communications Technical College \\ Wuhan, China
}

\author{
DENG Guang-hong \\ Department of Scientific Research on System \\ Wuhan Digital Engineering Institute \\ Wuhan, China
}

\begin{abstract}
The traditional random network coding methods with fixed multicast capacity which is difficult to get with the DTN dynamical features of mobility, intermittent connectivity and dynamic topology. Direct at this problem, a Dynamic Random Network Coding (DRNC) method used in Delay Tolerant Networks (DTN) environment is proposed. According to the receiving data states of nodes, it first obtains the transmit speeds of every network channel, and then constructs the network flow graphic with channel capacities and calculates the network multicast capacity. Moreover, the random network coding scenario is extended and tailored according to the multicast capacity to transmit data packets in DTN environment. Based on this method, it can increase the data delivery probability and reduce the average latency of data packets. Ultimately, a simulated DTN environment is constructed and some simulation experiments are taken, the results show that, compare with the traditional network coding method with fixed multicast rate, the DRNC method has reduced the data delivery delay and increased the data delivery ratio.
\end{abstract}

Keywords-delay tolerant network; random network coding; multicast rate; multicast capacity

\section{INTRODUCTION}

Delay Tolerant Network (DTN) is proposed to work out the problem of interconnection in heterogeneous and unstable networks, which is a new type of network model. Compare with the traditional network, the DTN network has some typical dynamic characteristics: 1) dynamic link; 2) dynamic topology; 3) delay tolerant, since the intermittent connectivity between nodes, the data delivery delay in DTN network is often unpredictable and long time.

Network coding can be used in DTN to improve the network throughput, reduce the transmission delay, enhance the network robustness etc[1 3]. Ahlswede et al[1] have shown that network coding can achieve max flow of the single-source multicast network, while the traditional routing technology cannot attain generally. LI S Y R et al [2] also pointed out that linear network coding can achieve the multicast capacity of general multicast network.

But in the DTN environment, network topology, multicast rate always changed dynamically, which makes the traditional random network coding method become more difficult to put into use. First of all, the variant channel will transmit data with different multicast rates, if using the deterministic network coding method[4], it must design different encoding schemes for different multicast rates, which is not only increased the amount of calculation, but also occupied a large amount of space to save coding coefficients with different multicast rates[5,6]; secondly, the dynamic network topology make it extremely difficult to obtain global network topology information[7-9], and thus it is difficult to obtain the network multicast capacity[10-12]. But aim to get the network throughput as much as possible, the multicast rate should be equal to the multicast capacity of the network [5].

For the sake of improving the data delivery ratio and reduce the data delivery delay of network coding transmission in DTN environment, a dynamic random network coding method is proposed in this paper. It first obtains the channel capacity, constructed the network flow graph, and calculates the network multicast capacity; and then expands and tailors the random net-work coding scheme dynamically. Ultimately, a random network coding transmission algorithm with variable multicast rate which matched the network multicast capacity is designed, to dynamic encode and transmit in DTN environment using network coding. After these, simulations scene are designed and carried out on the ONE environment. The epidemic routing method, the static random network coding method and the dynamic random network coding method presented in this paper are compared and analyzed. The simulation results show that, the dynamic random network coding method achieved better data delivery performance in DTN network environment.

\section{MULTICAST CAPACITY CALCULATION}

In order to obtain the best network coding transmission efficiency, the source multicast rate should be equal to the network multicast capacity as near as possible [5]. For this, it first computed the multicast capacity of network, by steps of channel rate computing, network flow graph constructing, and multicast capacity calculating and forecasting. After that, the random network coding scheme can be adjusted dynamically according to the multicast capacity.

\section{A. The finite state Markov channel (FSMC) model}

The finite-state Markov channel (FSMC)[13,14] divided the variation of signal to noise ratio(SNR) into a limited number of states:

$$
\begin{gathered}
S=\left\{s_{1}, s_{2}, \cdots, s_{n}\right\}, s_{k} \in\left[\Gamma_{k}, \Gamma_{k+1}\right),(0 \leqslant k \leqslant n) \\
-\infty<\Gamma_{i}<\Gamma_{j}<\infty, 0 \leqslant i \leqslant j \leqslant n+1
\end{gathered}
$$


$\Gamma_{k+1}-\Gamma_{k}$ denotes the SNR range of each channel state, and assumed that the channel is the slow fading channel, it only changed between the adjacent state, as shown in Fig.1. The transition probability between the channel states is

$$
P_{i, j}=\mathrm{P}_{\mathrm{r}}\left\{S_{t+1}=j \mid S_{t}=i\right\},|i-j| \leqslant 1
$$

$S_{t}=i$ represents that the channel state is $i$ at time $t, P_{i, j}$ is the probability of channel state changed from $i$ to $j$.
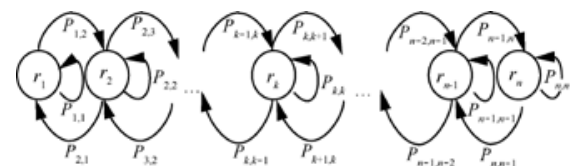

Fig.1The state transition diagram of the finite state Markov channel model

As in [14] show, in the Rayleigh fading channel, when the node $i$ receiving data, the probability density function of the instantaneous signal-to-noise ratio (SNR) $\Upsilon$ is

$$
p_{i}(r)=\frac{1}{\bar{r}_{i}} \exp \left(\frac{r}{\bar{r}_{i}}\right)
$$

$\overline{\Upsilon_{i}}=E\left(\Upsilon_{i}\right)$ is the average SNR, the steady-state probability when the channel at the state ${ }^{S_{k}}$ is

$$
\eta_{k}^{i}=\int_{\Gamma_{k}}^{\Gamma_{k+1}} p_{i}(r) d r
$$

The steady-state probability vector of the node i's channel state is $P_{\eta}^{i}=\left(\eta_{0}^{i}, \eta_{1}^{i}, \cdots, \eta_{n}^{i}\right)$, the state transition probability [13] of node $i$ is

$$
\begin{array}{r}
P_{k, k+1}^{i}=\frac{N\left(\Gamma_{k+1}\right) T_{c}}{\eta_{k}}, k=0,1,2, \cdots, n-1 \\
P_{k, k-1}^{i}=\frac{N\left(\Gamma_{k-1}\right) T_{c}}{\eta_{k}}, k=1,2, \cdots, n
\end{array}
$$

$N(\Gamma)$ is the cross function

$$
N(\Gamma)=\sqrt{\frac{2 \pi \Gamma}{r_{i}}} f_{d} \exp \left(-\frac{\Gamma}{\bar{r}_{i}}\right)
$$

$f_{d}$ is the Doppler frequency shift, if the symbol rate is constant, $T_{c}$ is a constant value [4]. The ${ }^{P_{k, k}^{i}}$ is

$$
P_{k, k}^{i}=\left\{\begin{array}{c}
1-P_{k, k-1}^{i}-P_{k, k+1}^{i}, 0<k<n \\
1-P_{0,1}^{i}, k=0 \\
1-P_{n, n-1}^{i}, k=n
\end{array}\right.
$$

So the state transition matrix of node $i$ is

$$
\boldsymbol{P}_{i}=\left[\begin{array}{cccccc}
P_{0,0}^{i} & P_{0,1}^{i} & \cdots & \cdots & \cdots & 0 \\
P_{1,0}^{i} & P_{1,1}^{i} & P_{1,2}^{i} & \cdots & \cdots & 0 \\
0 & \ddots & \ddots & \ddots & \ddots & 0 \\
\vdots & \ddots & \ddots & P_{n-1, n-2}^{i} & P_{n-1, n-1}^{i} & P_{n-1, n}^{i} \\
0 & \cdots & \cdots & \cdots & P_{n, n-1}^{i} & P_{n, n}^{i}
\end{array}\right]
$$

\section{B. Channel rate computing}

Assume that the channel state is stable in a time slot when receiving data, so in a time slot, the mathematic expectation of data amount received by node $i$ is

$$
\bar{D}=\omega\left(V \times L^{T}\right) P_{\eta}^{i T}
$$

$\omega$ is the consumed time slots when the source send a frame of data, $V=\left(v_{1}, v_{2}, \cdots, v_{n}\right)$ represents the corresponding rate of channel state, ${ }^{L}=\left(l_{1}, l_{2}, \cdots, l_{n}\right)$ represents the corresponding packet loss ratio of each channel rate, ${ }^{V \times L^{T}}=\left(v_{1} * l_{1}, v_{2} * l_{2}, \cdots, v_{n}{ }^{*} l_{n}\right)$. $P_{n}^{i \tau}$ represents the transpose of the channel steady-state probability vector.
Assume $D_{t}^{i}$ is node $i$ received the data numbers in a time slot, if $\alpha \bar{D}<D_{t}^{i}<\bar{D}$, it illustrates that the current channel is in a steady state, else it will switch states. $0<\alpha<1$, it's the adjustment coefficient, it can be set according to the test value.

The state vector of channel e is $S_{e}=\left(s_{1}, s_{2}, \cdots, s_{j} \cdots, s_{n}\right)$, the state elements values of channel state ${ }^{S_{k}}$ is:

$$
S_{j}=\left\{\begin{array}{l}
0, j \neq k \\
1, j=k
\end{array}, 1 \leqslant j \leqslant n\right.
$$

$S_{e}=\left(s_{1}, S_{2}, \cdots, S_{j} \cdots, S_{n}\right)$ is the state vector, chose the $k$ column of matrix $S_{e} \times P_{i}$ and transpose it.

$$
S P=\left[\left(S_{e} \times P_{i}\right)[][k]\right]^{\mathrm{T}}
$$

Find the maximum value of elements in the column, and set the value to 1 , other values are set to 0 , denoted as $S P^{\prime}$, so the new state vector of channel e is $S_{e}^{\prime}=S P^{\prime}$, correlate the channel state vector to the channel rate vector, we can get the current channel rate.

By using the above channel rate calculation method, the channel capacity of the each link (the same as channel rate; denote as $w_{i j}$ of the network flow graph) in the network can be obtained.

\section{Multicast capacity calculation}

Each channel capacity ${ }^{w_{i j}}$ was calculated in front section, and feedback to the source node through the feedback link, so the network flow graph at a moment can be get at the source node.

$$
A=\left[\begin{array}{ccccc}
w_{1,1} & w_{1,2} & \cdots & w_{1, n-1} & w_{1, n} \\
w_{2,1} & w_{2,2} & \cdots & w_{2, n-1} & w_{2, n} \\
\vdots & \vdots & \ddots & \vdots & \vdots \\
w_{n-1,1} & w_{n-1,2} & \cdots & w_{n-1, n-1} & w_{n-1, n} \\
w_{n, 1} & w_{n, 2} & \cdots & w_{n, n-1} & w_{n, n}
\end{array}\right]
$$

$w_{i j}$ is the channel rate from the node $i$ to $j, w_{i j}=0$ indicates that it's disconnect between $i$ and $j$.

For the network flow graph ${ }^{G}=(V, E), i \in V$, the set of out link started from node $i$ is called a partial cut of $i$, denoted as

$$
K_{i}=\left\{w_{i j},(i, j) \in E, i, j \in V\right\}
$$

The corresponding capacity matrix of the partial cut is.

$$
\boldsymbol{K}=\left[\begin{array}{ccccc}
K_{1,1} & K_{1,2} & \cdots & K_{1, n-1} & K_{1, n} \\
K_{2,1} & K_{2,2} & \cdots & K_{2, n-1} & K_{2, n} \\
\vdots & \vdots & \ddots & \vdots & \vdots \\
K_{n-1,1} & K_{n-1,2} & \cdots & K_{n-1, n-1} & K_{n-1, n} \\
K_{n, 1} & K_{n, 2} & \cdots & K_{n, n-1} & K_{n, n}
\end{array}\right]
$$

$K_{i, j}=\left\{w_{i m}, m \neq j,(i, m) \in E, i, j, m \in V\right\}$ denotes the set of out links from node $i$ but not to node $j$, it is the constrained partial cutting capacity. If there are no links between $i$ and $j$, then ${ }^{K_{i, j}}=\Lambda$.

Then ${ }^{C^{m}}=\bigcup_{i=1}^{m: 1} \bigcap_{j=1}^{m-1} K_{i j}$ is a cutting set of network $A$, According to the definition of the multicast capacity and the maximum flow and minimum cut theorem, the network multicast capacity $C$ can be get. 


\section{DYNAMIC EXPANSION OF THE RANDOM NETWORK CODING}

\section{A. Cropping and expansion of the random network coding scheme}

Assume the multicast rate of source node is $h$, and make it consistency with the multicast capacity, that is $h=C$. the broadcast data in a unit time is $x_{1}, x_{2}, \cdots, x_{h}$, each character are $m$ bits, the information encoding operation is in the field ${ }^{G F\left(2^{m}\right)}$. The encoded data transmitted by channel $e \in E$ is denoted as $I(e)$, it also belongs to the field of $G F\left(2^{m}\right)$. For the channel ${ }^{e=(i, j)}$, $i=\operatorname{tail}(e), j=\operatorname{head}(e)$.

In the random linear network coding, the output channel $e$ of each node $k, I(e)$ is denoted as:

$$
I(e)=\sum_{i=1}^{h} n_{e, i} x_{i}, \operatorname{tail}(e)=k
$$

Corresponding to the multicast rate $h$ and the coding vector $^{n_{e}=\left(n_{e, 1}, n_{\theta, 2}, \cdots, n_{e, j}\right)}$ of channel $e$, a random linear coding scheme $\Psi$ is denoted as.

$$
\Psi=\left\{\left(n_{1,1}, n_{1,2}, \cdots, n_{1, j}\right), \cdots,\left(n_{i, 1}, n_{i, 2}, \cdots, n_{i, j}\right)\right\} 0<i \leqslant E \mid, 0<j \leqslant h
$$

The $I(e)$ received by destination is the global encoded data which is times encoded by several relay nodes. The $I(e)$ received at the destination can be described as:

$$
I(e)=\sum_{i=1}^{h} g_{i} x_{i}
$$

$g_{i}=\boldsymbol{n}_{e} M_{k}, \boldsymbol{n}_{e}$ is a $1 X_{h}$ encoding vector matrix, ${ }^{\boldsymbol{M}_{k}}$ is the input matrix formed by the input vector of node $k$, it is matrix whose row number is the input channel number of node $k$, and column number is $h$.

For a coding scheme ${ }^{\Psi}$ with multicast rate is $h$ and a coding scheme ${ }^{\psi}$ with $k, k \leqslant h$, there is relationship below:

$$
\psi=\left\{n_{e, i}, e \in E, 1<i \leqslant k\right\}=\left\{\begin{array}{c}
\left\{n_{e, i} \in \Psi, 1<i \leqslant k\right\}, \operatorname{head}(e)=s \\
\Psi, \operatorname{head}(e) \neq s
\end{array}\right.
$$

It meaning that: as the output channel e of the source node $\mathrm{s}$, the code vector of channel $e$ in the scheme ${ }^{\psi}$ is composed of the front $k$ sub values of scheme $\Psi$ at the source node; for the other channels, the local encoding vectors in $\psi$ is the same as the $\Psi$, PU B X et al. [15] had proved this method is feasible.

Similarly, the coding scheme $\Psi$ can be obtained by adding $h-k$ encoding vectors as extension. As shown in Fig.2, $\Psi$ can be extended from ${ }^{\psi}$.

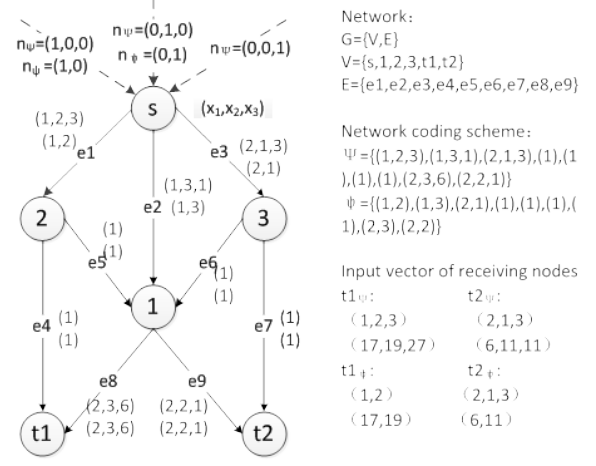

Fig.2 The extended example of the random network coding scheme

\section{B. The dynamic random network coding algorithm orient variable multicast rate}

By using the network multicast rate calculation method and the cutting and expansion method of random coding scheme, a dynamic random network coding algorithm orient variable multicast rate is designed in this paper. First, it takes an initial multicast rate to multicast, and using the random network coding method transmits to destination, then according to the link state information feedback by nodes, it adjusts the multicast rate and amends the encoding vector when next group transmission, and then re-adjust, re-amend and transfer, until finished the data transfer process. Algorithm description is shown as follow.

Input:

$G=(V, E):$ Network flow graph.

Steps :

1.if(source node)

2. Set the initial multicast rate $h$;

3. divide the data packet into group $x_{1}, x_{2}, \cdots, x_{h}$, and transmit them to adjacent node;

4. while (channel rate change)

5. calculate the multicast capacity $C$,set multicast rate $k=C$;

7. divide the data packet into group ${ }^{x_{1}, x_{2}, \ldots, x_{k}}$ and transmit them to relay nodes;

8. end while

9. end if

10.if(relay node)

11. receive data packet and acquire the network coding vector length $k$, compare it with $h$.

12. $\operatorname{if}(h=0)$

13. generated vector ${ }^{\left(n_{i, 1}, n_{i, 2}, \cdots, n_{i, k}\right)}$ for each output channel, construct coding scheme ${ }^{\Psi=\left\{\left(n_{1,1}, n_{1,2}, \cdots, n_{1, k}\right), \cdots,\left(n_{i, 1}, n_{i, 2}, \cdots, n_{i, k}\right)\right\}}$;

14. else if $(k<h)$

15. choose the first $k$ sub-value of the original coding scheme to generate a group coding scheme $\Psi=\left\{\left(n_{1,1}, n_{1,2}, \cdots, n_{1, \lambda}\right), \cdots,\left(n_{i, 1}, n_{i, 2}, \cdots, n_{i, k}\right)\right\} ;$

16. else if $(k>h)$

17. the relay nodes randomly generate $k$ - $h$ sub-value, and generate the random network coding scheme

18. end if

19. do network coding operation on input data by formula (10), and transmit them to output channels.

20. obtain the channel rate and feedback to the source node;

21. end if

22. if(destination node)

23. receive data packets, and construct network coding vector matrix ;

24. if(the matrix is full rank)

25. decode data by the formula (11);

26. end if

27. end if

\section{SIMULATION AND ANALYSIS}

In the simulation environment, it did simulations for three methods: transmitting with Epidemic Routing (ER), Static Random Network Coding (SRNC) and Dynamic Random Network Coding (DRNC) method proposed in this paper, and 
analyzed data delivery ratio and delivery delays of three methods.

The Fig.3 and Fig.4 is the delivery performance comparative of three methods with the node number changes in the ONE (the Opportunistic Network Environment simulator)[16] simulation environment.

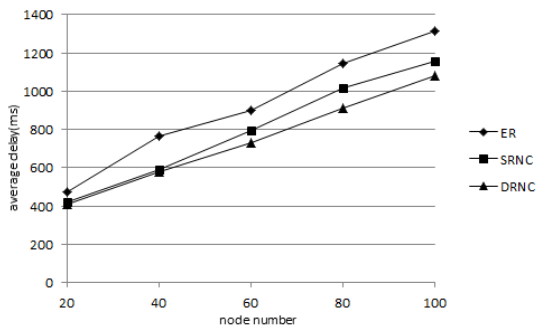

Fig.3 performance comparison of data delivery delay with node number changes

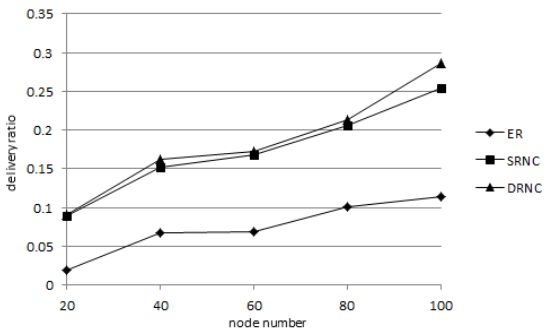

Fig.4 performance comparison of data delivery ratio with node number changes

Aim to verify the data delivery performance of the three methods in the situation that network topology and channel state change severely. It intensifies the variation of network topology and channel state by speeding up the moving speed of terminal nodes. The Fig. 5 and Fig. 6 make comparative analysis of the delivery delay and data delivery ratio of ER, SRNC and DRNC along with node speed changes in 100 nodes network.

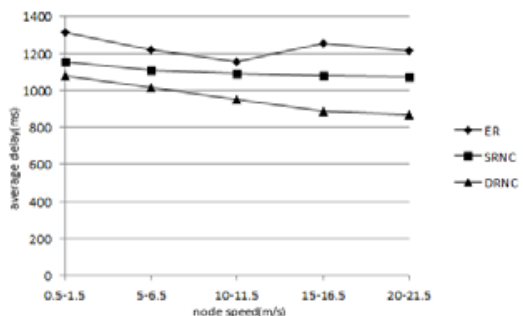

Fig.5 performance comparison of data delivery delay with node speed changes

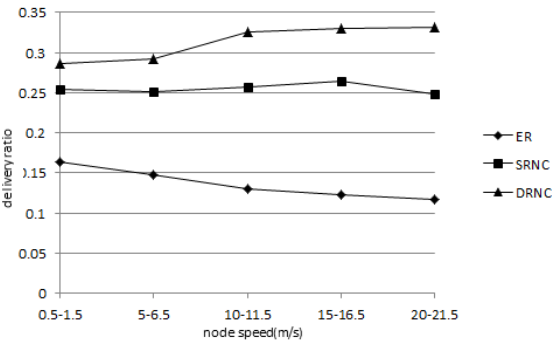

Fig.6 performance comparison of data delivery ratio with node speed changes

As the simulations results show, we can see that the DRNC method is more suitable for DTN network, in the conditions that the network size increased and node moving speed accelerated; the DRNC method has better data delivery performance.

\section{CONCLUSION}

Direct at the shortage of static random network coding method in DTN environment, it proposed a dynamic random network coding method in this paper. It calculates the network multicast capacity, and then designs the dynamic random network coding transmission method with variable multicast rate according to the multicast capacity. Finally, some simulations are carried out in DTN simulation environment ONE, and compare with other methods. The simulation results show that the DRNC method can gets better data delivery performance.

\section{References}

[1] AHLSWEDE R, NING C, LI S,et al. Network information flow[J]. IEEE Transactions on Information Theory. 2000,46(4): 1204-1216.

[2] LI S Y R, YEUNG R W, CAI N. Linear network coding[J]. IEEE Transactions on Information Theory, 2003,49(2): 371-381.

[3] TRACEY HO, M'EDARD M, KOETTER R, et al. A random linear network coding approach to multicast[J]. IEEE Transactions on Information Theory. 2006, 52(10):4413-4430

[4] LI B C, NIU D. Random network coding in Peer-to-Peer networks: from theory to practice[J]. Proceedings of the IEEE. 2011,99(3):513-523.

[5] HAEUPLER B, KARGER D R. Faster information dissemination in dynamic networks via network coding[A]. PODC'11[C].California, USA. 2011:381-390.

[6] FONG SL, YEUNG RW. Variable-rate linear network coding[J]. IEEE Trans on Information Theory. 2010,56(6):2618-2625.

[7] LU J, WU C K, XIAO S, et al. Efficient broadcast transmission algorithms based on opportunistic network coding[J]. Journal on Communications. 2012,33(1): 64-70.

[8] S ROUAYHEB A EL, SPRINTSON A, GEORGHIADES C. Robust network codes for unicast connections: a case study[J]. IEEE/ACM Transactions on Networking. 2011,19(3): 644-656.

[9] DEB S, MEDARD M, CHOUTE C. Algebraic gossip: a network coding approach to optimal multiple rumor mongering[J]. IEEE Transactions on Information Theory. 2006, 52(6): 2486-2507.

[10] PU B X, YANG L M, WANG W P. A deterministic data transmission approach with network coding under unknown network topology[J]. Acta Electronica Sinica, 2009, 37(10):2119-2124.

[11] WANG Y, LU H C, HONG P L, et al. Random linear network coding based alternative scheduling algorithms with two sources[J]. Journal of Electronics \& Information Technology. 2011,33(12): 3008-3014.

[12] GOPINATHAN A, PENG Z. Optimal layered multicast[J]. ACM Transactions on Multimedia Computing, Communications and Applications. 2011,7(2): 1-20

[13] ZHANG Q, KASSAM S. A finite-state markov model for rayleigh fading channels[J]. IEEE Transactions on Communications. 1999, 47(11):1688-1692.

[14] RAZAVILAR J, LIU K J R, MARCUS S I. Jointly optimized bitrate/delay control policy for wireless packet networks with fading channels[J]. IEEE Transactions on Communications. 2002, 50(3):484494.

[15] PU B X, YANG L M, WANG W P. Generation and extension of linear network coding[J]. Journal of Software. 2011,22(3): 558-571.

[16] KERÄNEN A, OTT J, KÄRKKÄINEN T. The ONE simulator for DTN protocol evaluation[A]. Proc of the 2nd International Conference on Simulation Tools and Techniques[C]. Rome, Italy, 2009. 